Originalien

Chirurg 2021 · 92:567-572

https://doi.org/10.1007/s00104-020-01281-w Online publiziert: 18 . September 2020

(c) Der/die Autor(en) 2020

Elisa Haucke ${ }^{1}$ Katharina Clever ${ }^{1}$ Stefan Watzke ${ }^{3}$ Johanna Schubert ${ }^{1}$.

Dietrich Stoevesandt ${ }^{1}$. Christiane Ludwig ${ }^{1}$ Sebastian Plößl ${ }^{2} \cdot$ Stefan K. Plontke ${ }^{2}$

' Dorothea-Erxleben-Lernzentrum, Medizinische Fakultät, Martin-Luther-Universität Halle-Wittenberg, Halle (Saale), Deutschland

${ }^{2}$ Universitätsklinik und Poliklinik für Hals-Nasen-Ohren-Heilkunde, Kopf- und Hals-Chirurgie, Universitätsklinikum Halle, Halle (Saale), Deutschland

${ }^{3}$ Klinik und Poliklinik für Psychiatrie, Psychotherapie und Psychosomatik, Universitätsklinikum Halle, Halle (Saale), Deutschland

\title{
„Hallesche OP-Wochen“: Wie ein Lehrformat das Interesse von Medizinstudierenden an der Chirurgie weckt
}

\begin{abstract}
Medizinstudierende messen den chirurgischen Fertigkeiten und dem Verständnis für die chirurgischen Prinzipien eine große Bedeutung für ihre spätere Tätigkeit zu. Die Ausbildung in diesen Fertigkeiten wird jedoch als inadäquat eingeschätzt [1]. Erhebungen unter Medizinstudierenden prognostizieren, dass am Ende des Studiums nur ca. $10 \%$ der Studierenden eine chirurgische Weiterbildung anstreben [7]. Die vorliegende Studie zeigt, dass das Lehrformat "Hallesche OP-Wochen" das Verständnis für chirurgische Abläufe verbessert und die Einstellung gegenüber chirurgischen Fachgebieten positiv beeinflusst.
\end{abstract}

\section{Hintergrund und Fragestellung}

Eine studierendenorientierte Ausbildung ist in bestimmten klinischen Bereichen schwierig oder nur unzureichend umsetzbar, da Zeit- und personeller Mangel den Klinikalltag prägen $[9,10]$. Aufgrund begrenzter räumlicher Kapazitäten und Hygienevorschriften ergeben sich zudem Einschränkungen für die praktische Ausbildung im Operationssaal. Neue Lehrkonzepte sind notwendig, um mit den gegebenen Umständen eine qualitativ hochwertige, praktisch orientierte und studierendenzentrierte Ausbildung zu gewährleisten und für die Chirurgie zu begeistern.

Die Liveübertragung oder Videoaufzeichnung chirurgischer Eingriffe ist durch den Fortschritt der Technik eine immer beliebtere Methode, um die operative Medizin abzubilden. Oft kommt dieses Lehrformat allerdings erst in der Weiterbildung zum/r Facharzt/ärztin zum Einsatz und umfasst wenige Lehreinheiten aus einzelnen Fachbereichen $[4,5,8]$. Endoskopien, Laparoskopien und roboterassistierte Operationen werden dabei häufig angeboten, da sich die Eingriffe technisch komplikationslos übertragen lassen [2].

Die „Halleschen OP-Wochen“ stellen ein Novum in der Liveübertragung realer Operationen und chirurgischer
Eingriffe für Studierende dar. In einer zweiwöchigen Veranstaltung präsentieren verschiedene chirurgische Fachrichtungen des Universitätsklinikums Halle (UKH) „klassische“, d.h. für das chirurgische Fachgebiet typische und häufig durchgeführte Operationen oder Interventionen im Bereich der Inneren Medizin oder interventionellen Radiologie. Die geplanten Eingriffe werden im Vorfeld online bekannt gegeben, um den Studierenden die Möglichkeit zu geben, sich mit den jeweiligen Operationstechniken und Krankheitsbildern vertraut zu machen. Neben der moderierten Liveübertragung der Operation in Hörsäle wird durch ergänzende Hintergrundinformationen vertiefend auf das jeweilige Krankheitsbild einschließlich Anatomie,

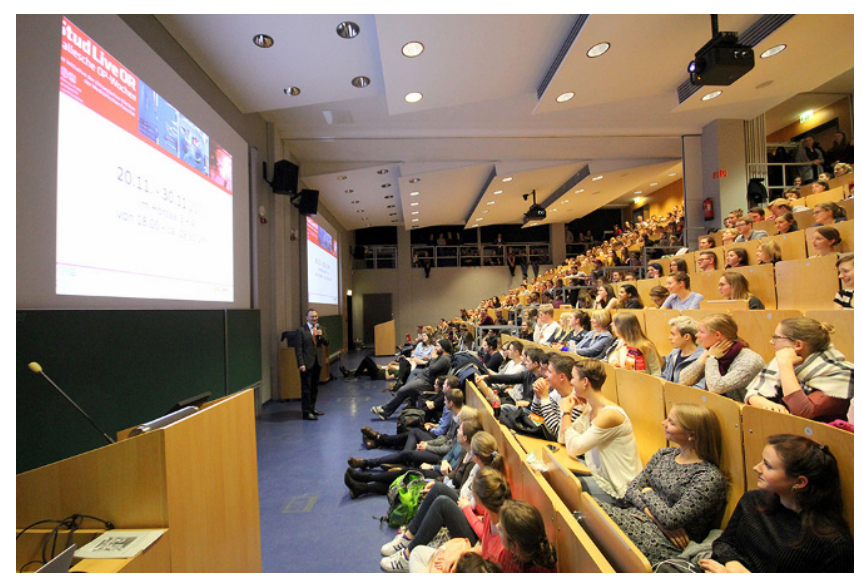

Abb. $1<$ Liveübertragung von Operationen in miteinander verbundene Hörsäle 
Tab. 1 Chirurgische Fachgebiete mit den jeweils demonstrierten Operationen

\begin{tabular}{|c|c|c|c|}
\hline Tag & Fachgebiet & Operation & (n) \\
\hline 1 & Allgemeinchirurgie & Cholezystektomie & 48 \\
\hline 2 & Orthopädie & Knieendoprothese & 63 \\
\hline 3 & Neurochirurgie & Vestibularisschwannom & 110 \\
\hline 4 & Hals-Nasen-Ohren-Heilkunde & Cochleaimplantat & 93 \\
\hline 5 & Traumatologie & Ersatzplastik vorderes Kreuzband & 83 \\
\hline 6 & Gynäkologie & Laparoskopische Hysterektomie & 76 \\
\hline 7 & Herz-Thorax-Chirurgie & Bypassoperation & 150 \\
\hline \multirow[t]{2}{*}{8} & Urologie & Tumornephrektomie & 86 \\
\hline & & & 709 \\
\hline
\end{tabular}

(Patho-)Physiologie und das Operationsverfahren eingegangen. Damit wird neben der curricularen Lehre (Blockpraktikum, Seminar und Vorlesung) unterstützend ein Teil der im Nationalen Kompetenzbasierten Lernzielkatalog Medizin (NKLM) festgelegten Lernziele abgebildet (Kap. 16: Therapeutische Prinzipien, Kap. 21: Erkrankungsbezogene Prävention, Diagnostik, Therapie, Versorgungs- und Notfallmanagement) und die Möglichkeit geboten, häufige Therapieverfahren kennenzulernen. In den curricularen Lehrveranstaltungen ist dafür nur begrenzt Zeit zur Verfügung und vor allem den Famulaturen/dem praktischen Jahr vorbehalten.

Die vorliegende Studie untersuchte, inwieweit sich die „Halleschen OP-Wochen “ eignen, das Interesse von Medizinstudierenden an chirurgischen Fächern zu steigern. Dafür wurden der selbstberichtete Lerneffekt und die Einstellung der Studierenden gegenüber den einzelnen chirurgischen Fachgebieten untersucht. Um die Akzeptanz des Lehrformates zu evaluieren, wurden darüber hinaus die Bewertung von Struktur und Organisation der Veranstaltung analysiert und Teilnehmendenzahlen ausgewertet.

\section{Studiendesign und Unter- suchungsmethoden}

\section{Ablauf der "Halleschen OP- Wochen"}

Im Regelbetrieb der einzelnen Kliniken des UKHs gibt es an unterschiedlichen Tagen der Woche sog. „lange Tische“ für komplexe und zeitintensive Operationen mit einem zugeteilten Operationsteam. Im Rahmen der „Halleschen OP-Wochen" wird dies genutzt, damit an acht Abenden (jeweils 18:00 bis 20:00 Uhr über zwei Wochen) acht ausgewählte chirurgische Fachrichtungen den Studierenden eine für ihr Fachgebiet typische Operation vorstellen können (• Tab. 1).

Die Teilnahme war für die Studierenden fakultativ. Die Information über die „Halleschen OP-Wochen“ wurde über die Lehrveranstaltungen, die Fachschaft Medizin und die sozialen Netzwerke der Studierenden der Universitätsmedizin Halle verbreitet. Vor jeder Operation wurde das schriftliche Einverständnis des/der Patienten/in für die Liveübertragung eingeholt. Im Operationssaal wurden zusätzlich zu den je nach Fachrichtung und Operation verwendeten Kamerasystemen (Operationsmikroskope und Endoskope) allgemeine Saalkameras installiert. Der/die Operateur/in wurde mit einem Headset ausgestattet. Die visuelle und verbale Liveübertragung der Operationen erfolgte in bis zu vier miteinander verbundene Hörsäle des UKH (• Abb. 1).

Im Hörsaal wurden die Operationen von einem/r erfahrenen Chirurgen/in des jeweiligen Fachgebietes als Moderator/in begleitet, fachlich eingebettet und erläutert. Dies erfolgte in freiwilliger Unterstützung außerhalb der Dienstzeit. Der Ablauf der Veranstaltung war wie folgt gegliedert:

1. Fallvorstellung,

2. generelle Aspekte zum Krankheitsbild und zu den Therapieprinzipien (z.B. Kernpunkte für Prüfungen/ Staatsexamen/die ärztliche Praxis),
3. Beschreibung der Anatomie und der Operationstechnik,

4. Liveübertragung aus dem Operationssaal.

Während der Operation erfolgte eine Erklärung der einzelnen Arbeitsschritte durch den/die Operateur/in. Die Studierenden konnten live Fragen stellen, die entweder von den Moderatoren/ innen im Hörsaal oder dem/der Operateur/in direkt aus dem Operationssaal beantwortet wurden.

\section{Datenerhebung}

Im ersten Jahr der „Halleschen OP-Wochen“ (2014) erfolgte vor und nach jeder einzelnen Operation eine quantitative, anonymisierte Datenerhebung mittels selbstkonstruierter Fragebögen. Für die Zuordnung der Prä- und Post-Fragebögen einer Person generierten die Teilnehmenden einen 8-stelligen Teilnahmecode.

Neben soziodemografischen Angaben (Alter, Geschlecht, aktuelles Studienjahr) wurde die Beurteilung der (1) Struktur und Organisation der Veranstaltung auf einer 6-stufigen Skala im Schulnotensystem von $1=$,,sehr gut“ bis $6=$ „ungenügend“ erfragt (Post-Fragebogen). Auf einer 5-stufigen Skala $(1=$,trifft völlig“ $\mathrm{zu}, 5=$, trifft gar nicht

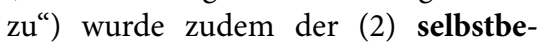
richtete Lerneffekt der Studierenden durch die folgenden Aussagen erfasst (Post-Fragebogen): ${ }^{\star}$ Die Veranstaltung war geeignet, mein Verständnis für den Ablauf der Operation zu verbessern und ${ }^{*}$ Die Veranstaltung war geeignet, mein Verständnis der zugrunde liegenden Erkrankung zu verbessern.

Zur Erfassung der (3) Einstellung zu den jeweiligen chirurgischen Fachgebieten bewerteten die Studierenden (Prä- und Post-Fragebogen) die folgenden Aussagen auf einer 5-stufigen Skala ( $1=$ „trifft völlig zu“, $5=$ „,trifft gar nicht zu“) am Beispiel der Allgemeinchirurgie: ${ }^{*}$ Allgemeinchirurgie finde ich spannend, ${ }^{*}$ Ich kann mir eine Famulatur in der Allgemeinchirurgie gut vorstellen und ${ }^{\star} I c h$ kann mir Allgemeinchirurgie als späteres Fachgebiet gut vorstellen. Die Aussagen 
wurden jeden Tag für das jeweilige chirurgische Fachgebiet neu formuliert.

\section{Datenanalyse}

Die Auswertung der Fragebögen erfolgte mithilfe des Statistikprogramms IBM SPSS Statistics (Version 25). Ein $\alpha-\mathrm{Ni}$ veau von 0,05 wurde für alle Berechnungen festgelegt. Charakteristika der Stichprobe, Struktur und Organisation der Veranstaltung, selbstberichteter Lerneffekt sowie Einstellung zur Chirurgie bzw. $\mathrm{zu}$ chirurgischen Fachgebieten wurden mithilfe deskriptiver Analysen (Häufigkeiten, Mittelwerte, Standardabweichungen) ausgewertet. Einstellungsänderungen in Bezug auf einzelne chirurgische Fachgebiete wurden anhand des $t$-Tests für abhängige Stichproben ausgewertet.

\section{Stichprobe}

Während der „Halleschen OP-Wochen“ wurden insgesamt 955 Prä- und 983 PostFragebögen ausgefüllt. In die Auswertung eingeschlossen wurden nur Medizinstudierende mit vollständig ausgefülltem Teilnehmercode und mindestens einer vollständigen Tagesevaluation (Präund Post-Fragebogen). Insgesamt lagen 709 vollständige Tagesevaluationen von 381 Studierenden vor, die in die vorliegende Auswertung eingehen. Mehr als $40 \%$ der Studierenden besuchten dabei mehr als eine Operation. $\bullet$ Tab. 1 zeigt die Anzahl der Tagesevaluationen pro Operation/Fachgebiet. Der Großteil der Studienteilnehmer/innen $(60,6 \% ; n=231)$ war zwischen 21 und 25 Jahren alt, 67,1\% waren weiblich, 32,9\% waren männlich. Studierende im 3. Studienjahr waren bei den „Halleschen OP-Wochen“ am häufigsten vertreten $(33,5 \% ; n=127)$.

\section{Ergebnisse}

\section{Struktur und Organisation}

Die Organisation der Veranstaltung, die Moderationen im Hörsaal und im Operationssaal, die technische Umsetzung sowie die Veranstaltung insgesamt wurden an den einzelnen Tagen von den Teilnehmenden von gut bis sehr gut bewertet. In - Tab. 2 sind die Bereiche angegeben, in

Chirurg 2021 - 92:567-572 https://doi.org/10.1007/s00104-020-01281-w

(c) Der/die Autor(en) 2020

E. Haucke · K. Clever · S. Watzke · J. Schubert · D. Stoevesandt · C. Ludwig · S. PlößI · S. K. Plontke

\section{"Hallesche OP-Wochen": Wie ein Lehrformat das Interesse von Medizinstudierenden an der Chirurgie weckt}

\section{Zusammenfassung}

Hintergrund. Eine studierendenorientierte Ausbildung ist in der praktischen Chirurgie schwierig und nur unzureichend umsetzbar. Neue Lehrkonzepte sind notwendig, um Studierenden die Chirurgie näherzubringen und sie für das Fach zu begeistern.

Methodik. In einer zweiwöchigen fakultativen Veranstaltung konnten Studierende der Humanmedizin insgesamt acht chirurgische Eingriffe aus verschiedenen Fachrichtungen live im Hörsaal mitverfolgen. Zeitgleich erfolgte im Hörsaal eine Moderation durch eine/n erfahrene/n Chirurgen/in. Anhand von Fragebögen (prä/post) wurde begleitend zu jeder Operation untersucht, inwieweit sich die Lehrveranstaltung eignet, das Verständnis und das Interesse für die chirurgische Tätigkeit zu verbessern.

Ergebnisse. Insgesamt lagen 709 vollständige Tagesevaluationen (prä und post) von 381 Studierenden vor. Der selbstberichtete Lerneffekt wurde von den Studierenden als gut bis sehr gut bewertet. In vielen dargestellten Fachgebieten zeigten sich durch die Teilnahme an den Liveoperationen signifikant positive Einstellungsänderungen in Bezug auf das jeweils operierende Fach allgemein, in Bezug auf eine geplante Famulatur und für die Wahl als späteres Fachgebiet.

Schlussfolgerung. Die hohen Teilnehmendenzahlen und die Evaluationsergebnisse lassen auf eine hohe Akzeptanz der Lehrveranstaltung schließen. Das Lehrformat ist geeignet, das Verständnis für chirurgische Abläufe zu verbessern sowie die Einstellung von Medizinstudierenden gegenüber chirurgischen Fachgebieten positiv zu beeinflussen.

Schlüsselwörter

Lehrmethoden · Liveoperation · LiveTeaching · Medizinstudium · Chirurgische Ausbildung

\section{"Halle surgery week": how a teaching format awakens medical students' interest in surgery}

Abstract

Background. A practical education in surgery is difficult to conduct in a student-centered manner and is thus often inadequate. New teaching concepts are required to provide students with insights into surgery and make the field more appealing.

Methods. As part of a two-week facultative event, medical students followed eight live surgical procedures from different disciplines from an auditorium. In the auditorium, the procedures were simultaneously moderated by an experienced surgeon. Before and after every procedure, questionnaires were used to analyze whether the teaching event was suited to improve an understanding and interest for the field of surgery.

Results. A total of 709 completed questionnaires (pre and post) from 381 students were collected. The self-reported learning effect was evaluated as good or very good by students. In many of the presented disciplines, experiencing a live surgical procedure was associated with significant positive changes in attitude regarding each discipline in general, a potential clinical traineeship in the discipline and choosing the discipline as a later specialization.

Conclusion. The high attendance as well as the evaluation results suggest a high acceptance for the teaching event. The teaching format is suited to improve the understanding of surgical procedures and had a positive effect on medical students' attitude towards surgical disciplines.

\section{Keywords}

Teaching methods - Live surgery - Live teaching · Medical studies · Surgical training 
Tab. 2 Bewertung zur Struktur und Organisation der "Halleschen OP-Wochen" ( $n=381)$

Welche Schulnote würden Sie...

MW-Bereich

SD-Bereich

der Organisation der Veranstaltung geben?

$1,3-1,7$

$0,5-0,8$

der technischen Umsetzung der Veranstaltung geben?

$1,4-2,5$

$0,6-1,0$

dem Vortragstil des Vortragenden im Hörsaal geben?

$1,2-1,9$

$0,4-0,9$

den Erläuterungen des Operateurs geben?

$1,1-2,3$

$0,3-0,9$

der Veranstaltung insgesamt geben?

$1,3-1,8$

$0,5-0,7$

Skala von 1 („sehr gut") bis 6 („ungenügend")

MW Mittelwert, SD Standardabweichung

denen sich die Mittelwerte der einzelnen Tagesevaluationen bewegen.

\section{Selbstberichteter Lerneffekt}

Die Teilnehmenden bewerteten die Veranstaltung überwiegend als gut bis sehr gut geeignet, den Ablauf einer Operation besser zu verstehen (•Tab. 3). Besonders gut bewertet wurden die Cholezystektomie, die Tumornephrektomie, das Cochleaimplantat und die Bypassoperation. Die Teilnehmenden gaben darüber hinaus an, dass die Veranstaltung gut geeignet war, um das Verständnis für die zugrunde liegende Erkrankung zu verbessern. Bei der laparoskopischen Hysterektomie zeigten weibliche Studierende dabei eine signifikant höhere Zustimmung zur Aussage „Die Veranstaltung war geeignet, mein Verständnis der zugrunde liegenden Erkrankung zu verbessern“ als männliche Studierende $(t(73)=-2,11$; $p<0,05)$.

\section{Einstellung zu einzelnen chirurgischen Fachgebieten}

Im Prä-Post-Vergleich zeigte sich, dass die Teilnahme an den Liveoperationen in einzelnen chirurgischen Fachgebieten signifikante Einstellungsänderungen bewirkte (• Tab.4). Die Bewertung der Aussage „Dieses Fachgebiet finde ich spannend" zeigte für die Fachgebiete HalsNasen-Ohren-Heilkunde, Herz-ThoraxChirurgie und Urologie signifikant positive Veränderungen.

In fünf der acht gezeigten Fachrichtungen (Allgemeinchirurgie, Neurochirurgie, Hals-Nasen-Ohren-Heilkunde und Urologie) zeigte sich ein signifikant positiver Effekt auf die Einstellung bezüglich des Interesses an einer Famulatur. Für die Allgemeinchirurgie, die
Orthopädie, die Neurochirurgie und die Urologie zeigte sich darüber hinaus eine signifikant positive Einstellungsänderung bezüglich der potenziellen Wahl als späteres Fachgebiet.

Hinsichtlich der Einstellung der Studierenden gegenüber den Fachrichtungen zeigten sich sowohl im Prä- als auch im Post-Fragebogen für die Orthopädie und die Gynäkologie signifikante Geschlechterunterschiede. Männliche Studierende bewerteten (post) die Orthopädie als späteres Fachgebiet signifikant besser als weibliche Studierende $(t(59)=2,73 ; p<0,01)$. Weibliche Studierende bewerteten (post) die Gynäkologie signifikant besser als männliche Studierende: spannendes Fach $(t(80)=-3,28 ; p<0,01)$, Famulatur $(t(81)=-2,53 ; p<0,05)$ und späteres Fachgebiet $(t(79)=-3,12 ; p<0,01)$. Für die anderen Fachgebiete wurden keine signifikanten Geschlechterunterschiede beobachtet.

\section{Diskussion}

Bei den „Halleschen OP-Wochen“ wurden acht Operationen aus verschiedenen chirurgischen Fachrichtungen gezeigt, an denen insgesamt mindestens 381 Studierende der Humanmedizin fakultativ teilnahmen. Mehr als 40 \% der Studierenden besuchten dabei mehr als eine Operation. Die Struktur und Organisation der Veranstaltung wurden insgesamt als (sehr) gut bewertet. Die hohen Studierendenzahlen und die gute Evaluation des Veranstaltungsformates lassen auf eine hohe Akzeptanz der Lehrveranstaltung sowie allgemein auf ein großes Interesse an der Chirurgie schließen. Der selbstberichtete Lerneffekt wurde von den Studierenden als (sehr) gut empfunden, vor allem wenn es darum ging, den Ablauf einer Opera- tion $\mathrm{zu}$ begreifen. Eine Studie belegte, dass Videoübertragungen von Operationen mit paralleler Moderation Studierenden stärker die Möglichkeit bieten, eigene Fragen $\mathrm{zu}$ stellen sowie Fragen beantwortet $\mathrm{zu}$ bekommen, als die direkte Begleitung im Operationssaal [6]. Darüber hinaus wird der Lerneffekt einer solchen Veranstaltung als besser eingestuft im Vergleich zur herkömmlichen Lehre im Operationssaal [3]. Die Vermittlung ärztlicher und evidenzbasierter Handlungskompetenzen ist durch die Reform des Medizinstudiums zu kompetenzorientierten, integrierten Curricula von grundlegender Bedeutung. Liveübertragungen von Operationen im $\mathrm{Me}$ dizinstudium stellen eine gute Möglichkeit dar, Studierenden komplexes medizinisches Arbeiten näherzubringen. Die im NKLM (Kap. 16) angeführten Lernziele, operative Eingriffe beschreiben zu können, können durch das Miterleben von Operationen möglicherweise besser erreicht werden. Durch die Kameraführung haben Studierende einen guten Einblick in das Operationsgebiet, was häufig in den Operationssälen mit einer Gruppe von Studierenden schlecht abbildbar ist. Studierende erleben das Miteinander im Team, den Umgang mit Komplikationen und werden durch die zusätzliche Moderation vertiefend über das jeweilige Krankheitsbild sowie das Operationsverfahren informiert.

Die Evaluationsergebnisse zeigen, dass die präsentierten chirurgischen Fachrichtungen insgesamt als spannend bewertet werden. Darüber hinaus sind Famulaturen für die Studierenden in den jeweiligen Fachgebieten denkbar. Die Aussage ${ }^{*}$ Ich kann mir [Fachgebiet] als späteres Fachgebiet gut vorstellen erhielt bei allen Fachbereichen nur eine mittlere Zustimmung. Da fast $60 \%$ der teilnehmenden Studierenden aus dem 1. bis 3. Studienjahr stammten, ist die Wahl des späteren Fachgebiets bei vielen wahrscheinlich noch mit großer Unsicherheit verbunden.

Das Miterleben der Liveoperation hatte einen positiven Effekt auf die Einstellung der Studierenden bezüglich der einzelnen chirurgischen Fachgebiete. Für fünf Fachgebiete konnte durch die Teilnahme an den „Halleschen OP-Wochen“ 


\begin{tabular}{|c|c|c|c|c|}
\hline \multicolumn{3}{|c|}{ Die Veranstaltung war geeignet, mein Verständnis... } & \multirow{2}{*}{$\begin{array}{l}\text {... für den Ablauf der Operation zu } \\
\text { verbessern. } \\
\text { MW } \pm \text { SD }\end{array}$} & \multirow{2}{*}{$\begin{array}{l}\text {... der zugrunde liegenden Erkrankung } \\
\text { zu verbessern. } \\
M W \pm S D\end{array}$} \\
\hline Fachgebiet & (n) & Operation & & \\
\hline$A C$ & 48 & Cholezystektomie & $1,2 \pm 0,5$ & $2,0 \pm 1,0$ \\
\hline Ortho & 63 & Knieendoprothese & $1,5 \pm 0,6$ & $2,2 \pm 0,9$ \\
\hline Neuro & 110 & Vestibularisschwannom & $1,7 \pm 0,9$ & $2,0 \pm 0,9$ \\
\hline HNO & 93 & Cochleaimplantat & $1,4 \pm 0,6$ & $2,0 \pm 0,9$ \\
\hline UC & 83 & $\begin{array}{l}\text { Ersatzplastik vorderes } \\
\text { Kreuzband }\end{array}$ & $1,8 \pm 0,9$ & $2,1 \pm 0,9$ \\
\hline Gyn & 76 & $\begin{array}{l}\text { Laparoskopische Hysterek- } \\
\text { tomie }\end{array}$ & $2,1 \pm 0,9$ & $2,5 \pm 1,0$ \\
\hline HTC & 150 & Bypassoperation & $1,4 \pm 0,6$ & $2,3 \pm 0,9$ \\
\hline Uro & 86 & Tumornephrektomie & $1,3 \pm 0,5$ & $2,1 \pm 0,9$ \\
\hline
\end{tabular}

Tab. 4 Einstellungsänderungen in den einzelnen Fachgebieten

\begin{tabular}{|c|c|c|c|c|c|c|c|c|c|c|}
\hline \multirow[t]{2}{*}{ Fachgebiet } & & \multicolumn{3}{|c|}{ Spannendes Fach } & \multicolumn{3}{|l|}{ Famulatur } & \multicolumn{3}{|c|}{ Späteres Fachgebiet } \\
\hline & & $M W \pm S D$ & $T$ & $p$ & $M W \pm S D$ & $T$ & $p$ & $M W \pm S D$ & $T$ & $p$ \\
\hline \multirow[t]{2}{*}{$\mathrm{AC}(n=48)$} & Prä & $1,90 \pm 0,79$ & \multirow[t]{2}{*}{1,35} & \multirow[t]{2}{*}{0,18} & $2,30 \pm 1,21$ & \multirow[t]{2}{*}{2,21} & \multirow[t]{2}{*}{$<0,05$} & $3,35 \pm 1,02$ & \multirow[t]{2}{*}{2,46} & \multirow[t]{2}{*}{$<0,05$} \\
\hline & Post & $1,83 \pm 0,81$ & & & $2,17 \pm 1,10$ & & & $3,20 \pm 1,05$ & & \\
\hline \multirow{2}{*}{$\begin{array}{l}\text { Ortho } \\
(n=56)\end{array}$} & Prä & $2,43 \pm 1,09$ & \multirow[t]{2}{*}{1,30} & \multirow[t]{2}{*}{0,20} & $2,69 \pm 1,28$ & \multirow[t]{2}{*}{1,55} & \multirow[t]{2}{*}{1,28} & $3,48 \pm 1,26$ & \multirow[t]{2}{*}{2,19} & \multirow[t]{2}{*}{$<0,05$} \\
\hline & Post & $2,34 \pm 1,12$ & & & $2,56 \pm 1,26$ & & & $3,35 \pm 1,30$ & & \\
\hline \multirow{2}{*}{$\begin{array}{l}\text { Neuro } \\
(n=104)\end{array}$} & Prä & $1,81 \pm 0,81$ & \multirow[t]{2}{*}{1,82} & \multirow[t]{2}{*}{0,07} & $2,85 \pm 1,21$ & \multirow[t]{2}{*}{2,24} & \multirow[t]{2}{*}{$<0,05$} & $3,75 \pm 1,12$ & \multirow[t]{2}{*}{1,99} & \multirow[t]{2}{*}{$<0,05$} \\
\hline & Post & $1,72 \pm 0,86$ & & & $2,72 \pm 1,25$ & & & $3,66 \pm 1,19$ & & \\
\hline \multirow{2}{*}{$\begin{array}{l}\text { HNO } \\
(n=90)\end{array}$} & Prä & $2,47 \pm 1,13$ & \multirow[t]{2}{*}{3,95} & \multirow[t]{2}{*}{$<0,001$} & $2,72 \pm 1,30$ & \multirow[t]{2}{*}{3,06} & \multirow[t]{2}{*}{$<0,01$} & $3,33 \pm 1,34$ & \multirow[t]{2}{*}{1,06} & \multirow[t]{2}{*}{0,29} \\
\hline & Post & $2,22 \pm 1,12$ & & & $2,53 \pm 1,28$ & & & $3,27 \pm 1,35$ & & \\
\hline \multirow[t]{2}{*}{ UC $(n=78)$} & Prä & $1,69 \pm 1,02$ & \multirow[t]{2}{*}{$-1,00$} & \multirow[t]{2}{*}{0,32} & $1,95 \pm 1,18$ & 0,00 & 1,00 & $2,91 \pm 1,28$ & $-1,27$ & 0,21 \\
\hline & Post & $1,74 \pm 0,96$ & & & $1,95 \pm 1,20$ & & & $2,96 \pm 1,26$ & & \\
\hline Gyn & Prä & $2,45 \pm 1,09$ & $-0,55$ & 0,58 & $2,75 \pm 1,28$ & $-0,16$ & 0,87 & $3,51 \pm 1,35$ & 0,50 & 0,62 \\
\hline$(n=74)$ & Post & $2,49 \pm 1,07$ & & & $2,76 \pm 1,35$ & & & $3,48 \pm 1,33$ & & \\
\hline & Prä & $1,50 \pm 0,65$ & 2,91 & $<0,01$ & $2,22 \pm 1,13$ & 3,14 & $<0,01$ & $3,18 \pm 1,12$ & 2,52 & $<0,05$ \\
\hline$(n=143)$ & Post & $1,41 \pm 0,64$ & & & $2,08 \pm 1,14$ & & & $3,07 \pm 1,30$ & & \\
\hline Uro $(n=85)$ & Prä & $2,36 \pm 0,96$ & 3,49 & $<0,01$ & $2,76 \pm 1,30$ & 3,30 & $<0,01$ & $3,59 \pm 1,30$ & 3,19 & $<0,01$ \\
\hline & Post & $2,15 \pm 0,98$ & & & $2,54 \pm 1,27$ & & & $3,39 \pm 1,28$ & & \\
\hline
\end{tabular}

eine positive Einstellungsveränderung in Bezug auf die Wahl als späteres Fachgebiet beobachtet werden, fünf Fachgebiete wurden in der Post-Befragung als spannender bewertet und in drei Fachgebieten zeigte sich eine positive Einstellungsveränderung in Bezug auf das Interesse an einer Famulatur.

Die Teilnahme am Lehrformat ist für die Studierenden fakultativ, sodass Selektionseffekte in der Stichprobe nicht ausgeschlossen werden können. Chi- rurgisch interessierte Studierende sind möglicherweise insgesamt überrepräsentiert. Chirurgische Vorerfahrungen der Studierenden (z.B. durch Lehrveranstaltungen, Famulaturen/Praktika) wurden nicht erfasst, hatten sich jedoch gegebenenfalls bereits vor der Teilnahme an den „Halleschen OP-Wochen“ positiv auf die Einstellung der Studierenden zu den chirurgischen Fachgebieten ausgewirkt. Da die Studierenden größtenteils mit guten bis sehr guten Bewertung einstiegen, ist zudem eine zusätzliche Verbesserung nur begrenzt möglich.

Das übergeordnete Ziel, das Interesse von Medizinstudierenden für die Fächer der Chirurgie zu steigern, konnte durch die „Halleschen OP-Wochen“ erreicht werden. Zum einen hat die Veranstaltung „Leuchtturmcharakter", was eine gesteigerte Aufmerksamkeit am Fach Chirurgie zur Folge hat. Zum anderen weckt das große Engagement der Chirurgen/innen und die ausführlichen Darstel- 
lungen und Erläuterungen zu operativen Abläufen das Interesse der Studierenden und motiviert ebenso, Famulaturen in einzelnen chirurgischen Fächern zu erwägen. Das breite Angebot, an verschiedenen Fachrichtungen und die Größe der Veranstaltung, d.h. die Möglichkeit mehrere Operationen anzusehen, rechtfertigt die Ausrichtung als mehrtägiges Format.

\section{Ausblick}

Für die Studierenden der Medizinischen Fakultät an der Martin-Luther-Universität Halle-Wittenberg finden die „Halleschen OP-Wochen“" mittlerweile jährlich statt. Es werden stetig neue Fachrichtungen, wie z.B. Dermatologie, Geburtshilfe, plastische Chirurgie, Augenheilkunde, Mund-Kiefer-Gesichts-Chirurgie und seit 2016 auch Interventionen der Inneren Medizin (z. B. Endoskopie) und der interventionellen Radiologie eingebunden, wodurch die Studierenden neue typische Operationstechniken und Interventionen, Krankheitsbilder und Therapiemöglichkeiten kennenlernen. Zudem können neben den Studierenden der Human- und Zahnmedizin auch Studierende der Gesundheitsund Pflegewissenschaften sowie Schüler/ innen des Ausbildungszentrums der Universitätsmedizin Halle - im Sinne einer interprofessionellen Ausbildung - an den „Halleschen OP-Wochen“ teilnehmen.

\section{Fazit für die Praxis}

Was Lehrformat der „Halleschen OP-
Wochen“ wird von den Studierenden
sehr gut angenommen und steigert
das Interesse von Studierenden an
einzelnen chirurgischen Fächern.
- Die Teilnahme an den „Halleschen
OP-Wochen“ (u. a. durch das Miter-
leben von Operationen; die Mode-
ration durch erfahrene Chirurgen/
innen und die Möglichkeit, aktiv
Fragen zu stellen) verbessert das
Verständnis der Medizinstudieren-
den für chirurgische Abläufe und
Operationsverfahren.
Die Vielfalt an dargebotenen chirur-
gischen Interventionen bietet allen
Medizinstudierenden die Möglich-

keit, einen breiten Einblick in die chirurgische Tätigkeit zu bekommen.

- Die Liveübertragung von Operationen für Studierende stellt eine sinnvolle Ergänzung der chirurgischen Ausbildung dar.

\section{Korrespondenzadresse}

\section{Prof. Dr. med. Stefan K. Plontke}

Universitätsklinik und Poliklinik für Hals-NasenOhren-Heilkunde, Kopf- und Hals-Chirurgie, Universitätsklinikum Halle

Ernst-Grube-Str. 40, 06120 Halle (Saale),

Deutschland

stefan.plontke@uk-halle.de

Funding. Open Access funding provided by Projekt DEAL.

\section{Einhaltung ethischer Richtlinien}

Interessenkonflikt. E. Haucke, K. Clever, S. Watzke, J. Schubert, D. Stoevesandt, C. Ludwig, S. Plößl und S.K. Plontke geben an, dass kein Interessenkonflikt besteht.

Für diesen Beitrag wurden von den Autoren/innen keine Studien an Menschen oder Tieren durchgeführt. Für die aufgeführten Studien gelten die jeweils dort angegebenen ethischen Richtlinien.

Open Access. Dieser Artikel wird unter der Creative Commons Namensnennung 4.0 International Lizenz veröffentlicht, welche die Nutzung, Vervielfältigung, Bearbeitung, Verbreitung und Wiedergabe in jeglichem Medium und Format erlaubt, sofern Sie den/die ursprünglichen Autor(en) und die Quelle ordnungsgemäßnennen, einen Link zur Creative Commons Lizenz beifügen und angeben, ob Änderungen vorgenommen wurden.

Die in diesem Artikel enthaltenen Bilder und sonstiges Drittmaterial unterliegen ebenfalls der genannten Creative Commons Lizenz, sofern sich aus der Abbildungslegende nichts anderes ergibt. Sofern das betreffende Material nicht unter der genannten Creative Commons Lizenz steht und die betreffende Handlung nicht nach gesetzlichen Vorschriften erlaubt ist, ist für die oben aufgeführten Weiterverwendungen des Materials die Einwilligung des jeweiligen Rechteinhabers einzuholen.

Weitere Details zur Lizenz entnehmen Sie bitte der Lizenzinformation auf http://creativecommons.org/ licenses/by/4.0/deed.de.

\section{Literatur}

1. Birch DW, Mavis B (2006) A needs assessment study of undergraduate surgical education. Can J Surg 49(5):335-340

2. Brunckhorst $\mathrm{O}$, Challacombe $\mathrm{B}$, Abboudi $\mathrm{H}$ et al (2014) Systematic review of live surgical demonstrations and their effectiveness on training. Br J Surg 101(13):1637-1643

3. Gul YA, Wan ACT, Darzi A (1999) Undergraduate surgical teaching utilizing telemedicine. Med Educ 33(8):596-599

4. Lenihan J, Brower M (2012) Web-connected surgery: using the internet for teaching and proctoring of live robotic surgeries. J Robot Surg 6(1):47-52

5. McCarthy PM (2010) Going live: implementing a telesurgery program. AORN J 92(5):544-552

6. McIntyre TP, Monahan TS, Villegas L et al (2008) Teleconferencing surgery enhances effective communication and enriches medical education. Surg Laparosc Endosc Percutan Tech 18(1):45-48

7. Osenberg D, Huenges B, Klock M et al (2010) Wer wird denn noch Chirurg? Chirurg 6:308-315

8. Roser F, Pfister G, Tatagiba M et al (2013) Live surgery in neurosurgical training courses: essentia infrastructure and technical set-up. Acta Neurochir 155(3):541-545

9. Rüsseler M, Weber R, Braunbeck A et al (2010) Training praktischer Fertigkeiten in der Chirurgie Ein Ausbildungskonzept fürStudierende.Zentralbl Chir 135(3):249-256

10. Schürer S, Schellberg D, Schmidt J et al (2006) Evaluation der traditionellen studentischen Ausbildung in der Chirurgie. Eine Heidelberger Bestandsaufnahme. Chirurg 77(4):352-359 